\title{
An Exploration of Community Detection and Recommendation Systems (CDR) in Social Networks
}

\author{
Dr. T. Venu Gopal ${ }^{1}$, Mantri Charan Babu' ${ }^{2}$ Dr. K. Kranthi Kumar ${ }^{3}$ \\ ${ }^{1}$ Professor, Department of CSE, JNTUHCEJ, Nachupalli, Karimnagar, Telangana, INDIA, t_vgopal@rediffmail.com \\ ${ }^{2}$ Asst. Professor, Dept of CSE, Sreenidhi Institute of Science and Technology, Hyderabad, INDIA, mcharan32@ gmail.com \\ ${ }^{3}$ Assoc. Professor, Dept of IT, Sreenidhi Institute of Science and Technology, Hyderabad, INDIA, drkkranthikumar@gmail.com \\ Received Date : October 03, 2021 Accepted Date : October 25, 2021 Published Date : October 07, 2021
}

\begin{abstract}
Community detection and Recommender systems are assumed as significant parts in helping the web users discover important information by proposing information of likely interest to them and a central task for network analysis means to segment a network into numerous substructures to assist with uncovering their inactive capacities. Community detection has been widely concentrated in and extensively applied to numerous real world network problems. Because of the possible worth of social relations in recommender systems, social recommendation has drawn in expanding consideration in recent years.
\end{abstract}

As the issues that network strategies attempt to solve and the network information to be determined become progressively more complex, new methodologies have been proposed and created, traditional ways to deal with community detection and recommendation commonly use probabilistic graphical models and implement an assortment of earlier information to deduce community structures. Regardless of all the new progression, there is as yet an absence of astute comprehension of the hypothetical and methodological supporting of local area location, which will be fundamentally significant for future advancement of the space of social network analysis.

In this paper, we start by giving conventional meanings of social networks terms and talk about the novel property of social networks and its implications. Unified architecture of network community finding methods to characterize the state-of-the-art of the field of community detection. In particular, we give a complete survey of the current community detection techniques and audit of existing recommender systems examine some exploration bearings to further develop social network capabilities.
Key Words : Complex Network, Community Detection, Social Recommender Systems, Social Network Analysis and Social Media.

\section{INTRODUCTION}

In this section we discusses introduction to community detection and social recommender systems in detail.

\subsection{Community Detection}

Network models, while they have a long history in sociological and software engineering research, are as of late discovering new applications in brain science. Customarily, a network is displayed utilizing a chart, and much network investigation depends on graph theory. A graph is made out of a bunch of nodes meaning the subjects being examined, and a set of edges mirroring the connections between the subjects. In traditional social network analysis, these nodes are individuals and the edges address relational connections, like friendships. A typical model is Zachary's Karate Club, as shown in Figure.1, a network of university karate club individuals where the edges demonstrate whether each pair of individuals is friends [1]. Examining social relations has its undeniable applications for social researchers considering individual and gathering conduct, yet the nodes are not restricted to addressing individuals.

There are numerous likely inquiries of interest when dissecting network information. The focal point of this paper is on one specifically: community detection. Community detection distinguishes groups of nodes inside networks that, as far as their neighbors, are inside associated yet remotely detached [2]. In the karate network example, there was a parted of the club individuals into two groups after a conflict. The objective of community detection here is to recognize who has a place with which group, in view of the 
individuals recorded friendships. The genuine split in the club is portrayed in the above Figure .1, as distinguished by the differentially shaded nodes.

Attending with the expanded utilization of network data is a developing measure of literature committed to the undertaking of accurately recognizing node enrollment in communities. While the condition of the science has progressed rapidly in a brief time frame, there are still gaps in the capacity of accessible community detection algorithms to give dependable outcomes. To be specific, most algorithms were intended to break down huge networks; be that as it may, there have been not many assessments of how the techniques act as far as really recuperating group structure like what has been led in the long history of conventional group investigation [3], [4], [5], [6], [7]. Moreover, it isn't certain whether these "adaptable" algorithms will demonstrate dependable or helpful for some lines of request found in psychological investigations that utilization more modest examples of people.

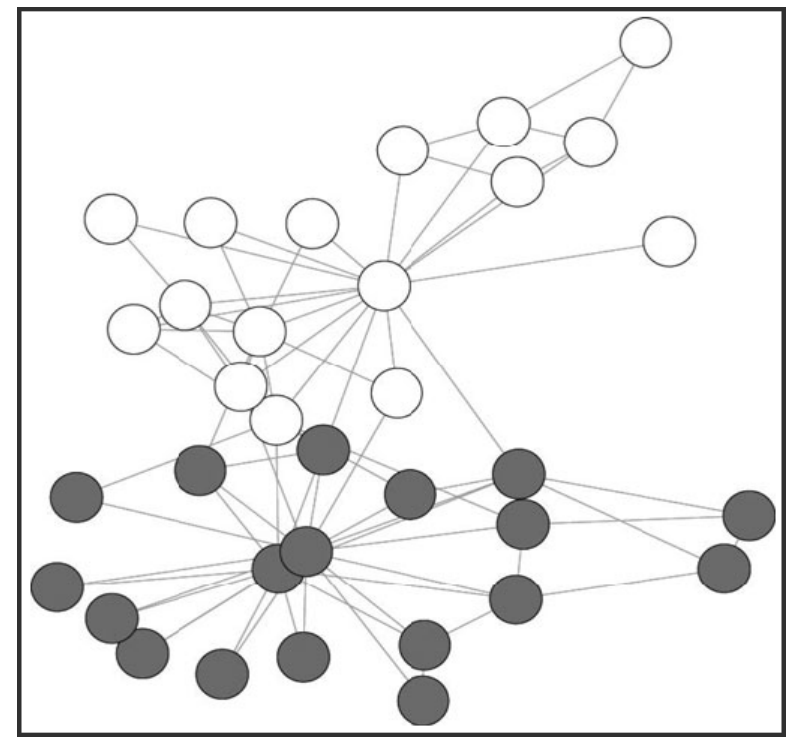

Figure 1: The Zachary's Karate Club network depicts friendships between 34 members of a collegiate karate club (Zachary, 1977). The nodes of this graph are colored to indicate group membership after the club split into two factions.

\subsection{Social Recommender}

With the improvement of the Internet, data has expanded at a phenomenal rate and the data over-burden issue has gotten progressively serious for online users. For instance, when we need to purchase a computer and search "computer" in Amazon, it returns 11,100,260 items. Recommender systems, which endeavor to handle the data over-burden issue by proposing data that is of possible interest to online users, have become significant and famous [8], [9], [10], [11], [12]. For information users, great suggestions permit them to rapidly discover applicable data covered in a lot of irrelevant data. For information suppliers, recommender systems not just assist with figuring out which data to present to individual buyers, yet in addition further develop purchaser dedication since buyers will in general revisitation of the destinations that best serve their necessities [13].

Recommender system turned into a free exploration region during the 1990s [14] and have drawn in much consideration from numerous disciplines, like mathematics, physical science, psychology, and software engineering [15]. Numerous strategies are utilized to fabricate recommender systems, which can be by and large characterized into content-based techniques collaborative filtering (CF) based strategies, and hybrid strategies [16]. Content-based strategies, established in information retrieval [17] and information filtering research [18], prescribe things like the ones the client liked before. CF-based techniques foresee client interests straight by revealing mind boggling and sudden examples from a client's previous practices and prescribe things to a client from different users with comparative interests and inclinations in the past [19], [20]. Hybrid strategies join content-based and CF-based techniques.

The increasing prevalence of online media incredibly enhances individuals social activities with their families, friends, and partners, which produces rich social relations like friends in Facebook, following in Twitter and trust relations in Epinions. Online social relations give an alternate method to people to impart carefully and permit online users to impart thoughts and insights with their associated users. A client's inclination is like or affected by their socially associated followers and the reasoning behind the suspicion can be clarified by friendly connection speculations, for example, homophily [21] and social impact [22]. Homophily shows that users with comparable inclinations are bound to be associated, and social impact uncovers that users who are associated are bound to have comparative inclinations.

\subsection{Preliminaries}

We initially present the terms and notations, and later present an order of the strategies for community detection that we will discuss in this paper.

\subsubsection{Definitions, Terms and Notations}

\section{A. Network}

A network $G=(V, E, X)$ consists of $n$ nodes $V=\left\{v_{1}, v_{2} \ldots\right.$ $\left.v_{n}\right\}, m$ edges $E=\left\{e_{i j}\right\} \subseteq V \times V$, and a maximal number $q$ of 
attributes $x_{i}$ on a node $v_{i}$, where all $x_{i}$ 's collectively give rise to an $n \times q$ attribute matrix $X=\left(x_{i}\right)_{n \times q}$. The topological structure of $G$ can be defined by an $n \times n$ adjacency matrix $A$ $=\left(a_{i j}\right)_{n \times n}$, where $a_{i j}=1$ if $e_{i j} \in E$, or 0 , otherwise. $G$ is undirected if

$a_{i j}=a_{j i}$, or directed, otherwise [23].

\section{B. Community}

The network $G$ contains $k$ communities $C=\left\{C_{1}, C_{2}, \ldots, C_{k}\right\}$, where $C_{i}$ is a subgraph of $G$ and the nodes within $C_{i}$ are densely connected whereas the nodes across $C_{i}$ and $C_{j}$ are sparsely connected. The communities are non-overlapping when $C_{i} \cap C_{j}=\emptyset \forall i, j$.

\section{Community Detection}

Given a network $G$, community detection is to design a mapping $F$ to assign every node $v_{i}$ of $G$ into at least one of the $k$ communities, i.e., to label $v_{i}$ at least one community identity $C_{i} \in\left\{C_{1}, C_{2}, \ldots, C_{k}\right\}$. Equivalently, the problem is to derive a community assignment of nodes $C=$ $\left(c_{1}, c_{2}, \ldots, c_{n}\right)$.

\section{Social Recommendation}

In a typical recommender system, there is a set of users and a set of items. Let $u=\left\{u_{1}, u_{2} \ldots u_{n}\right\}$ and $v=$ $\left\{v_{1}, v_{2} \ldots v_{m}\right\}$ be the sets of users and items respectively, where is the number of users and is the number of items. A user $u_{i}$ rates a subset of items with some scores. We use $\mathrm{R} \in$ $\mathrm{R}^{n \times m}$ to denote the rating matrix where $\mathrm{R}_{i j}$ is the rating score if $u_{i}$ gives a rating to $v_{j}$, otherwise we employ the symbol "?" to denote the unknown rating. Usually the rating matrix is very sparse, suggesting that there are lots of unknown ratings in $\mathrm{R}$. For example, the density of the rating matrix in commercial recommender systems is often less than $1 \%$ [12]. If item $v_{j}$ has attributes, we use $\mathrm{x}_{j} \in \mathrm{R}^{\ell}$ to represent $v_{j}$ where $\ell$ is the number of attributes. The task of recommender systems is to predict the rating for user $u_{i}$ on a non-rated item $v_{j}$ or to recommend some items for given users, i.e., to predict missing values in $\mathrm{R}$ based on known ratings. A short meaning of social proposal is any suggestion with online social relations as extra information, i.e., enlarging a current proposal motor with extra friendly signals. Social relations can be trust relations, friends or membership. The social recommender systems expect that users are associated when they set up friendly relations [10, $13,24]$.

The following sections of this paper are organized as follows. In Section 2 and 3, discusses various community Detection methods and Recommendation Systems and their existing work. Section 4, describes applications of CDR systems. Section 5 and 6 provides a clear path towards the future works/direction in the CDR system and finally in section 7 conclusion of our work.

\section{COMMUNITY DETECTION METHODS}

Various algorithms exist for discovering networks in network information. This paper surveys and looks at eight of the most conspicuous and available community detection algorithms. These techniques are a decent portrayal of the present status of the field - both in assortment and use. The eight algorithms remembered for this investigation are edge betweenness (EB), fast greedy (FG), walk-trap (WT), label propagation (LP), leading eigenvector (LE), multilevel community (ML), spin glass community (SG) and optimal community (OC). These algorithms are summed up beneath.

\subsection{Edge betweenness}

An early Community detection algorithm, now and again alluded to as Girvin and Newman's algorithm, is edge betweenness [24]. This is a troublesome algorithm that chips away at the suspicion that edges interfacing nodes of isolated networks will be given high centrality scores, where centrality is some estimation of the "significance" of a node inside its organization. Betweenness is an edge centrality measure determined by checking the quantity of most limited ways between each node pair that remembers the edge for question.

\subsection{Fast greedy}

The fast greedy algorithm is an agglomerative top-down level grouping technique that improves $\mathrm{Q}$ by combining the pair of networks at each progression to deliver the biggest expansion in Q [24] Thinking about an improved on recipe for $\mathrm{Q}$ in (1)

$$
Q=\sum_{k}\left(e_{k k^{\prime}}-e_{k}^{2}\right)
$$

Where $e_{k k}$ is the fraction of edges in the network that

connects $C_{k}$ and $C_{k^{\prime}}, e_{k k^{\prime}}=\frac{L_{C_{k}}}{L}$ and $e_{k}=\sum_{k^{\prime}} e_{k k^{\prime}}$ Then the changes in $\mathrm{Q}, \Delta Q$, When Two groups is as in (2)

$$
\Delta Q=2\left(e_{k k^{\prime}}-e_{k} e_{k^{\prime}}\right)
$$

Where $\Delta Q$ is modularity for every pair of communities.

\subsection{Walktrap}

The Walktrap method uses short random walks on a graph to detect communities [25]. It assumesthat random walks within a graph should get "trapped" within the communities. A random walk process begins on a selected node andmoves to another node chosen randomly and uniformly from its neighbors, and then proceeds to a next node in the same way, with the number of steps specified as the walk length. The length of the walk must be short enough to not be trivial, but long enough to gather community information. 


\subsection{Label propagation}

Label propagation starts with every node having a different name, and iteratively refreshes nodes to assume the names of most of its adjoining nodes [26]. Networks with high thickness will rapidly embrace a typical label. The cycle proceeds until all nodes have a place with the local area that the greatest number of its neighbors has a place with:

1. Every node starts with a different name.

2. In an irregular successive order, adjust the name of every node to be that of most of its neighbors (on account of no greater part, a mark is chosen arbitrarily).

3. Repeat step 2 until every node has the name of most of its neighbors for each node $i$.

\subsection{Leading eigenvector}

Newman developed another algorithm based on optimizing modularity and related to spectral clustering methods called Leading Eigenvector [27]. This method begins with the modularity matrix $\mathrm{B}=\left\{q_{i j}\right\}_{N \times N}$ where $q_{i j}=x_{i j}-2 L$. B is used in place of the Laplacian matrix of traditional spectral clustering. Considering the two cluster case, $\mathbf{p}$ is a vector partition of the graph where $\boldsymbol{p}_{\boldsymbol{i}}=1$ if $v_{i}$ belongs to the first cluster and $\boldsymbol{p}_{\boldsymbol{i}}=-1$ if it belongs to the second. If $\lambda \mathrm{j}$ is an Eigen value of $B$ with associated eigenvector $\mathrm{u} \mathrm{j}$ then modularity can be rewritten as in (3):

$$
B=\frac{1}{4 L} \sum_{i=1}^{N}\left(u_{i}^{T} \cdot p\right)^{2} \lambda_{i}
$$

Denoting the largest eigen value as $\lambda_{1}$, its associated eigenvector, $\mathbf{u}_{1}$, is selected as the best partition, grouping the nodes with their signs. B always has a trivial eigen- vector $\boldsymbol{u}_{\boldsymbol{i}}=(1,1, \ldots, 1)$ with corresponding eigen value $\lambda_{i}=0$. In the case where there is no community structure, there will be no positive Eigen values so the maximum is the trivial eigenvector where all nodes are part of one community.

\subsection{Multilevel community (Louvain method)}

Multilevel is one more greedy modularity maximization based methodology initially produced for weighted networks. The objective of the "multilevel community" algorithm is to find the high modularity divisions of the network without the resolution issues associated with greedy optimization of modularity [28]. This algorithm has a likely issue of neighborhood optima, on the grounds that the yield is subject to the request for the nodes being considered.

\subsection{Spin glass community}

Spin glass community is a strategy dependent on the Potts model, which emerges from the field of statistical mechanics. The technique made by [29] maps a network onto a zero-temperature K-Potts model with closest neighbor communications. The Potts model is an arrangement of spins that can be in $\mathrm{K}$ various states, streamlining an energy function. Associating this to community detection, the spin states are the gathering names of the nodes, and the energy of the spin framework is the quality function of the communities.

\section{8. Optimal community}

Optimal Community searches through all potential allotments of a diagram and picks the one that boosts measured quality. While this technique is vigorous, it is the most restricted as far as applications, as it isn't suggested for charts with more noteworthy than 50 nodes. This undertaking is $N P$-complete [28].

\section{RECOMMENDER SYSTEMS}

Collaborative Filtering (CF) is generally embraced to fabricate recommender systems, and most existing social recommender systems depend on CF procedures. Social recommendation has two data sources, i.e., a) rating data and b) social information. Most existing social recommender systems pick CF models as their fundamental models to fabricate systems and propose ways to deal with catch social data dependent on outcomes from social network analysis.

In this manner, an overall $\mathrm{CF}$ based social recommendation framework contains two sections:

1) A essential CF model and

2) A social data model, which can be officially expressed as in (4)

a social recommendation CF model $=$ a basic CF model + a social information model

The essential CF model in a social suggestion CF model makes an approach to order friendly recommender systems. Following the grouping of CF based recommender systems, we characterize social recommender systems into two significant classifications as indicated by their fundamental CF models:

1) Memory based social recommender systems

2) Model based social recommender systems.

\subsection{Memory based Social Recommender Systems}

Memory based social recommender systems use memory based CF models, particularly client situated techniques as their fundamental models. A missing rating for a given client is amassed from the ratings of users corresponded to her, signified as $\mathrm{N}^{+}$. For a given client, customary client arranged strategies utilize comparative users, while memory based social recommender systems utilize associated users $\mathrm{N}^{+}$got from both rating data and social data. 
Social recommender systems in this classification ordinarily follow two stages. In the first place, they acquire the corresponded users $\mathrm{N}^{+}(\mathrm{i})$ for a given user $\mathrm{u}_{\mathrm{i}}$, and second is the traditional last advance of memory based $\mathrm{CF}$ techniques aggregating from the associated users got by the initial step for missing ratings. Distinctive social recommender systems in this classification utilize various ways to deal with get corresponded users $\mathrm{N}^{+}$in the initial step.

\subsubsection{Social based Weight Mean}

For a given $u_{i}$, this strategy $[32,33]$ simply considers a $u_{i}$ 's directly connected users $\mathrm{F}(\mathrm{i})$ as the set of her correlated users $\mathrm{N}^{+}(\mathrm{i})$ (as in (5)),

$$
\mathrm{N}^{+}(\mathrm{i})=\left\{\mathrm{u}_{\mathrm{j}} \mid \mathrm{T}(\mathrm{i}, \mathrm{j})=1\right\}
$$

\subsubsection{TidalTrust}

Users in this framework [8] are associated through trust relations. The authors plan a metric TidalTrust to assess trust values among users dependent on the accompanying two perceptions:

1) Shorter propagation ways produce more exact trust estimates.

2) Paths with higher trust values make better outcomes. To estimate trust values among users.

\subsubsection{MoleTrust}

Another trust metric, MoleTrust [10, 34, 35] is proposed and comprises of two significant stages. To start with, cycles in trust networks are eliminated. To get trust values, an enormous number of trust spreads must be executed. Along these lines, eliminating trust cycles in advance from trust organizations can fundamentally accelerate the proposed algorithm in light of the fact that each client just should be visited once to surmise trust values. With this activity, the first trust network is changed into a directed non-cyclic graph.

Second, trust values are determined dependent on the acquired coordinated non-cyclic diagram by playing out a basic chart random walk: first the trust of the users at 1-hop away is registered, then, at that point the trust of the users at 2-hop away, and so on After trust values are processed, MoleTrust characterizes users inside maximum-depth and have appraised the objective thing as connected usersN ${ }^{+}$, where maximum-depth is a predefined parameter.

\subsubsection{TrustWalker}

The instinct of this framework [36] is from two key perceptions. Initial, a client's social organization has little cross-over with users like her [37], proposing that social data gives an autonomous wellspring of data. Second, appraisals from emphatically believed friends on comparative things are more solid than ratings from feebly confided in neighbors on a similar objective thing. The principal perception demonstrates the significance of trust based methodologies while the subsequent perception proposes the capacity of thing focused methodologies. To exploit the two methodologies, TrustWalker proposes an irregular walk model to join trust based and client situated methodologies into a reasonable structure. It inquiries a client's immediate and backhanded companion's ratings for the objective thing just as comparable things by performing arbitrary stroll in online social networks.

\subsection{Model based Social Recommender Systems}

Model-based social recommender systems pick modelbased CF strategies as their fundamental models. Network factorization procedures are broadly utilized in model based CF strategies. There are a few pleasant properties of these framework factorization methods $[38,39]$.

1. Numerous advancement techniques, for example, gradient based methods can be applied to track down an all-around worked ideal arrangement, scaled to a great many users with a large number of trust relations

2. Matrix factorization has a pleasant probabilistic interpretation with Gaussian noise

3. It is truly adaptable and permits us to incorporate earlier knowledge.

Most existing social recommender systems in this class depend on matrix factorization [11, 24, 40, 41, 42, 43, 44, $45,46,47,48,49,50,51]$. The normal reasoning behind these strategies is that client's inclinations are like or affected by users whom they are socially associated with. Nonetheless, the minimal expense of social connection arrangement can prompt social relations with heterogeneous qualities (e.g., feeble ties and solid ties combined as one) [52]. Since users with solid ties are bound to have comparable preferences than those with feeble ties, treating all friendly relations similarly is probably going to prompt debasement in suggestion execution. Subsequently for every friendly connection, these techniques partner a strength, which is typically determined by rating comparability in existing social recommender systems.

As per various meanings of Social $(T, S, \Omega)$, we further gap social recommender systems in this classification into three gatherings: 
T. Venu Gopal. et al., International Journal of Advances in Computer Science and Technology, 10(10), October 2021, 23 - 33

1. Co-factorization strategies

2. Group techniques

3. Regularization strategies.

\subsubsection{Co-factorization methods}

The underlying assumption of systems in this group [24, $46]$ is that the $\mathrm{i}^{\text {th }}$ user $\mathrm{u}_{\mathrm{i}}$ should share the same user preference vector $u_{i}$ in the rating space (rating information) and the social space (social information). Social recommender systems in this group perform a cofactorization in the user- item matrix and the user-user social relation matrix by sharing the same user preference latent factor. SoRec [24] and LOCABAL [46] are two representative systems in this group.

\subsubsection{Ensemble methods}

The essential thought of gathering strategies $[43,47]$ is that users and their social networks ought to have comparable ratings on things, and a missing rating for a given client is anticipated as a direct mix of appraisals from the client and her social organization. STE [43] and mTrust [47] are two representative systems.

\subsubsection{Regularization methods}

Regularization methods [11, 42] focus on a user's preference and assume that a user's preference should be similar to that of her social network. For a given user ui, regularization methods force her preference ui to be closer to that of users in ui's social network Ni. SocialMF [42] and Social Regularization [11] are two representative systems in this group.

\section{APPLICATIONS}

We start our conversation with a synopsis of genuine uses of community detection and Recommender systems in numerous application fields.

\subsection{Practical Applications}

We examine the utilizations of community detection and recommender systems on various areas.

\subsubsection{Applications in Different Areas}

Community detection and Recommender systems has assorted applications across various spaces like online social networks and neuro science. Online social networks, including Facebook, Twitter and Wechat, involve the interactions among individuals through the web. Finding people group in such organizations is a compelling method to surmise the connections of people, which has been taken on for undertakings like spammer detection and crisis response.
Jin et al. [56] demonstrate that connections in online social networks by and large convey semantic in-line, and networks of connections can more readily describe local area practices contrasted with networks of nodes. Taking into account this, they plan a clever probabilistic model investigating network geography and connection substance inside and out to perform interface local area location to viably mine social connections among people.

Wu et al. [57] plan an original end-to-end deep learning model, i.e., MRF with GCN, in view of GCN which straight forwardly works on coordinated informal organizations. They present in their model a MRF layer that catches client following data to refine forecast made by GCN for social spammer identification.

Neuroscience is a discipline considering the sensory systems and brain. With the new improvement of mind planning and neuro imaging strategies, the brain has started to be demonstrated as network. A lot of exertion has been advanced to take advantage of such networks to assist with removing the practical regions of the brain.

Liu et al. [58] propose a framework of Siamese community preserving graph convolutional network (SCP-GCN). The strategies initially holds the community structure considering the intra-community and inter community properties in learning process, and afterward utilizes Siamese design that models the pair-wise closeness to direct this learning system, in order to become familiar with the underlying and practical joint inserting of brain networks.

Jin et al. [59] contend that the current examinations normally build community structures of brain networks utilizing resting-state useful magnetic resonance imaging (fMRI) information, while overlooking the intrinsic planning and legitimacy of fMRI time series. They present the dynamic time warping (DTW) algorithm that investigates the synchronization and asynchronism of fMRI time series to remove the relationship between's brain regions.

\section{FUTURE WORKS IN COMMUNITY DETECTION}

While learning-based community detection, including probabilistic graphical model and deep learning, has shown predominant performance across an assortment of issues and areas, there are provokes that should be tended to. In this part, we momentarily talk about these difficulties and future exploration bearings conceivably worth pursuing. 


\subsection{Large Networks}

With the quickly expanding size of network information, all the more enormous organizations have turned into the norm across various logical spaces. These organizations regularly have many thousands or billions of nodes and edges just as perplexing underlying examples. Most existing community detection techniques face unreasonable requirements on such huge organizations because of the conceivably restrictive interest on memory and algorithm. They might require countless preparing cases or model boundaries to make the current techniques successful. Besides, the current methodologies normally handle these issues by network decrease or estimation, which might lose some significant organization data and influence the demonstrating exactness. This brings up the issue of how to devise an edge work that far surpasses the current benchmark approaches in accuracy and efficiency.

\subsection{Community Interpretability}

In spite the fact that community detection has been read for over 10 years, the interpretability of community stays a significant and basic issue to be enough tended to. Most current community identification techniques use highest level words or short expressions in the outcomes to sum up networks, despite the fact that the trait data of nodes is normally finished sentences that have more data than individual words. Notwithstanding, these techniques may not be natural enough for understanding the semantics of networks because of the modest number of words and muddled connection between words. The most effective method to utilize network data to give a superior semantic understanding to local area is one of future examination bearings.

\subsection{Adaptive Community Model Selection}

Adaptive model choice for community detection means to pick the most proper algorithm for finding community, as per the qualities of various networks or explicit necessities of various undertakings. Although the current techniques can be stretched out starting with one network or assignment then onto the next somewhat, not many of them think about how to perform model variation. In this way, center has moved to planning a brought together engineering that can consequently adjust to explicit undertakings or networks while keeping up with model exactness and strength as opposed to proposing assorted structures for various networks or assignments. This is an arising research region that would be testing however fulfilling.

\subsection{Networks with Complex Structures}

Many real world networks are heterogeneous, dynamic, hierarchical, or incomplete. Heterogeneous networks are those that contain different types of nodes and edges, or different types of descriptions on nodes and edges, such as text and images. Dynamic networks are networks whose topology and/or attributes change over time. Dynamic networks appear when nodes and edges are added or deleted, thus altering the properties of nodes or edges. Hierarchical networks are composed of several layers, each of which has specific semantics and functions. Incomplete networks are the ones with missing information of their topology, nodes, or edges. While these networks can be partly explored by the learning based community detection, there still exist several serious issues. First, most existing methods assume homogeneous networks, which may in fact difficult to handle. Second, due to the variability of dynamic networks, most existing methods, especially the ones based on deep learning, need to be re-trained over a series of steps when the networks evolve, which is very time consuming and may not meet the real time processing demand. Third, hierarchical networks typically have different types of relationships across the network hierarchies, which are important while often not well handled by the existing methods. Moreover, almost all the existing methods regard the networks to be analyzed to be complete and accurately documented without noise. Unfortunately, this is rarely the case in practice as it is challenging to obtain complete information of the networks. Therefore, new methods should be developed to handle these issues to better improve the performance of community detection on these types of complex networks.

\subsection{Integrating Statistical Modeling and Deep Learning} Although a few techniques have been proposed to join factual demonstrating with profound learning, like MRF as$\mathrm{GCN}$, it is as yet a virgin yet encouraging examination region. For example, the current techniques commonly use the earlier information that statistical model proposals to refine the embedding are of GCN to improve coming about communities. However, these strategies may not completely think about the time complexity or interpretability of the models, raising huge difficulties to community detection practically speaking. Besides, it stays an open issue to incorporate factual displaying in profound learning techniques.

\section{FUTURE WORKS IN SOCIAL RECOMMENDATION SYSTEM}

Since the exhibition help changes from one space to another, social recommendation is as yet in the beginning phases of 
improvement and a functioning space of investigation. In this subsection, we talk about a few exploration bearings that might conceivably work on the capacities of social recommender systems and make social recommendation appropriate to a considerably more extensive scope of uses.

\subsection{The Heterogeneity of Social Networks}

Most existing social recommender systems treat a user's connections homogeneously. However, connections in online social networks are intrinsically heterogeneous and are a composite of various types of relations $[47,59,60]$. Figure illustrates an example of $u_{1}$ 's social relations with $\left\{u_{2}, u_{3}, \ldots, u_{9}\right\}$. The user $u_{1}$ may treat her social relations differently in different domains. For example, $u_{1}$ may seek suggestions about "Sports" from $\left\{u_{2}, u_{3}\right\}$, but ask for recommendation about "Electronics" from $\left\{u_{4}, u_{5}\right\}$. In [47], the authors found that people place trust differently to users in different domains. For example, $u_{i}$ might trust $u_{j}$ in "Sports" but not trust $u_{j}$ in "Electronics" at all. For different sets of items, exploiting different types of social relations can potentially benefit existing social recommender systems [47].

\subsection{Weak Dependence Connections}

Most existing model-based social recommender systems exclusively utilize a client's solid reliance associations, i.e., direct connections, which disparage the variety of user's viewpoints and tastes [60]. In the event that users in the actual world just had solid reliance associations, life would be really exhausting since solid reliance associations demonstrate solid similarities. In reality, users can set up frail reliance associations with others in social networks when they are not straightforwardly associated. Frail reliance associations can give significant setting data about users' inclinations, and are demonstrated to be valuable in job hunting [61], the diffusion of ideas [61], knowledge transfer [64] and relational learning [62], while rarely used in recommendation.

\subsection{User Segmentation}

In conventional recommender systems, for a given client, ratings of users generally like her are totaled to anticipate a missing rating. At the point when engaged with social data, other than being comparable, users are socially associated. A client's most comparative users typically have little crossover with her associated users [37].

As per the quantity of pulled in ratings, things can be fragmented into cold-start things and ordinary things. Various sorts of users might contribute diversely for various kinds of things. For instance, associated users can further develop the proposal exactness of cold-start areas [65], while comparative users are critical to suggest normal items [34]. Accordingly, microcosmic examinations of users and things might give us a more profound comprehension of the job of social networks and might possibly further develop proposal execution.

\subsection{Temporal Information}

User inclinations for items float over the long run. For instance, individuals intrigued by "Gadgets" at time $\mathrm{t}$ might move their inclinations to "Sports" at time $t+1$. Transient data is a significant factor in recommender systems and there are conventional recommender systems that consider temporal information [58, 63]. Transient elements in information can essentially affect exactness than planning more complex learning algorithms [68].

\subsection{Negative Relations}

Right now most existing social recommender systems utilize positive relations, for example, friendships and trust relations. In any case, in online media, users additionally determine negative relations like doubt and abhorrence. Creators in [65] tracked down that negative relations are significantly more significant than positive relations, uncovering the significance of negative relations for social recommendation. There are a few works taking advantage of distrust $[32,66]$ in social recommender systems. They treat trust and doubt independently, and essentially use doubt in a contrary method to trust, for example, sifting questioned users or considering doubt relations as bad loads. Notwithstanding, trust and doubt are molded by various components of dependability, and trust influences conduct aims uniquely in contrast to distrust [67]. Besides, doubt relations are not autonomous of trust relations [33]. A more profound comprehension of negative relations and the connections to positive relations can assist us with creating effective social recommender systems by taking advantage of both positive and negative social relations.

\subsection{Cross Media Data}

A user by and large has different records in web-based media. For instance, a client who has a record in Epinions may likewise have a record in eBay. Another client on one site may have existed on one more site for quite a while. For instance, a client has as of now indicated her inclinations in Epinions and has additionally composed many audits about things. At the point when the client registers at eBay interestingly as a cool beginning client, information about the client in Epinions can assist eBay with taking care of the chilly beginning issue and precisely prescribe things to the client. Incorporating networks from numerous sites can achieve a colossal effect on friendly recommender systems 
and give a productive and successful approach to tackle the hard beginning issue. The principal trouble of incorporating information is associating relating users across sites and there is late work proposed to handle this mapping problem [68]. The investigation of the planning issue makes coordination of cross-media information for social suggestion conceivable and achieves new freedoms for social recommender systems.

\section{CONCLUSION}

In this article defined the terms like Network, Community, Community Detection and Social Recommendation. We discussed in detail about the different methods in both Community Detection and Recommendation Systems. Listed out the various applications in the CDR area and also described the future directions of the CDR with respect to various scope of work.

\section{REFERENCES}

1. Zachary, W. W. An information flow model for conflict and fission in small groups. Journal of Anthropological Research, 33(4), 452-473. DOI: 10.1086/jar.33.4.3629752. 1977.

2. Cormack, R. M. A review of classification. Journal of the Royal Statistical Society. Series A (General), 134(3), 321-367. DOI: 10.2307/2344237. 1971.

3. Milligan, G.W. An examination of the effect of six types of error perturbation on fifteen clustering algorithms. Psychometrika, 45(3), 325-342. DOI: 10.1007/BF02293907. 1980

4. Steinley, D. Local optima in K-means clustering: What you don't know may hurt you. Psychological Methods, 8(3), 294. DOI: https://doi.org/10.1037/1082989X.8.3.294. 2003

5. Steinley, D. K-means clustering: A half-century synthesis. British Journal of Mathematical and Statistical Psychology, 59(1), 1-34. DOI: 10.1348/000711005X48266. 2006

6. Steinley, D., \& Brusco, M. J. Initializing K-means batch clustering: A critical evaluation of several techniques. Journal of Classification, 24(1), 99-121. DOI: 10.1007/s00357-007-0003-0. 2007

7. Steinley, D., Brusco, M. J., \&Wasserman, S. Clusterwise $\mathbf{p} \square$ models for network data. Statistical Analysis and Data Mining, 4, 487-496. DOI: 10.1002/sam.10139. 2011

8. Golbeck, J.: Generating predictive movie recommendations from trust in social networks. Trust Management pp. 93-104 Springer, 2006.

9. Koren, Y.: Collaborative filtering with temporal dynamics. In: Proceedings of the $15^{\text {th }}$ ACM SIGKDD international conference on Knowledge discovery and data mining, pp. 447-456. ACM, 2009.

10. Massa, P., Avesani, P.: Trust-aware recommender systems. In: Proceedings of the 2007 ACM conference on Recommender systems, pp. 17-24. ACM, 2007.
11. Ma, H., Zhou, D., Liu, C., Lyu, M., King, I.: Recommender systems with social regularization. In: Proceedings of the fourth ACM international conference on Web search and data mining, pp. 287-296. ACM, 2011.

12. Sarwar, B., Karypis, G., Konstan, J., Riedl, J.: Itembased Collaborative filtering recommendation algorithms. In: Proceedings of the 10th international conference on World Wide Web, pp. 285-295. ACM, 2001.

13. Schafer, J.B., Konstan, J.A., Riedl, J.: E-commerce recommendation applications. Data mining and knowledge discovery 5(1), 115-153, 2001.

14. Ellenberg, J.: This psychologist might outsmart the math brains competing for the Netflix prize. Wired Magazine, March pp. 114-122, 2008.

15. Adomavicius, G., Tuzhilin, A.: Toward the next generation of recommender systems: A survey of the state-of-the-art and possible extensions. IEEE Transactions on Knowledge and Data Engineering, 17(6), 734-749, 2005.

16. Baeza-Yates, R., Ribeiro-Neto, B., et al.: Modern information retrieval, vol. 463. ACM press New York. 1999.

17. Belkin, N.J., Croft, W. B.: Information filtering and information retrieval: two sides of the same coin? Communications of the ACM 35(12), 29-38, 1992.

18. Koren, Y.: Factorization meets the neighborhood: a multifaceted collaborative filtering model. In: Proceeding of the 14th ACM SIGKDD international conference on Knowledge discovery and data mining, pp. 426-434. ACM, 2008.

19. Su, X., Khoshgoftaar, T.: A survey of collaborative filtering techniques. Advances in Artificial Intelligence 2009.

20. McPherson, M., Smith-Lovin, L., Cook, J.: Birds of a feather: Homophily in social networks. Annual review of sociology pp. 415-444, 2001.

21. Marsden, P., Friedkin, N.: Network studies of social influence. Sociological Methods and Research 22(1), 127-151, 1993.

22. F. D. Malliaros and M. Vazirgiannis, Clustering and community detection in directed networks: A survey, Phys. Rep.-Rev. Sec. Phys. Lett., vol. 533, no. 4, pp. 95-142, 2013.

23. Ma, H., Yang, H., Lyu, M., King, I.: Sorec: social recommendation using probabilistic matrix factorization. In: Proceeding of the 17th ACM conference on Information and knowledge management, pp. 931-940. ACM, 2008.

24. M. Newman and M. Girvan, Finding and evaluating community structure in networks, Phys. Rev. E, vol. 69, no. 2, p. 26113, 2004.

25. Pons, P., \& Latapy, M. Computing communities in large networks using random walks. In: Yolum, $\mathrm{P}$., Güngör, T., Gürgen, F., Özturan, C (Eds.), Computer and Information Sciences-ISCIS 2005 (pp. 284-293). Berlin, Germany: Springer. DOI: 10.1007/11569596_31.2005. 
T. Venu Gopal. et al., International Journal of Advances in Computer Science and Technology, 10(10), October 2021, 23 - 33

26. Raghavan, U. N., Albert, R., \& Kumara, S. Near linear time algorithm to detect community structures in largescale networks. Physical Review E, 76(3), 036106.

DOI: https://doi.org/10.1103/PhysRevE.76.036106. 2007.

27. Newman, M. E. Finding community structure in networks using the eigenvectors of matrices. Phys. Rev. E, vol. 74(3), no. 2, p. 26113. 2006.

28. Blondel, V. D., Guillaume, J. L., Lambiotte, R., \& Lefebvre, E. (2008). Fast unfolding of communities in large networks. Journal of Statistical Mechanics: Theory and Experiment, P10008. DOI: http://stacks.iop.org/1742-5468/2008/i=10/a=P10008. 2008.

29. Reichardt, J., \& Bornholdt, S. Statistical mechanics of community detection. Physical Review E, 74(1), 016110 . DOI: https://doi.org/10.1103/PhysRevE.74.016110. 2006.

30. Brandes, U., Delling, D., Gaertler, M., Gorke, R., Hoefer, M., Nikoloski, Z., \&Wagner, D. On modularity clustering. IEEE Transactions on Knowledge and Data Engineering, 20(2), 172-188. DOI: 10.1109/TKDE.2007.19068., 2008.

31. Victor, P., Cornelis, C., De Cock, M., Teredesai, A.M.: A comparative analysis of trust enhanced recommenders for controversial items. In: Proc. of the International AAI Conference on Weblogs and Social Media, pp. 342-345, 2009.

32. Victor, P., De Cock, M., Cornelis, C.: Trust and recommendations. In: Recommender Systems Handbook, pp. 645-675. Springer, 2011.

33. Massa, P., Avesani, P.: Trust-aware collaborative filtering for recommender systems. In: On the Move to Meaningful Internet Systems 2004: CoopIS, DOA, and ODBASE, pp. 492-508. Springer, 2004.

34. Massa, P., Avesani, P.: Controversial users demand local trust metrics: An experimental study on epinions.com community. In: Proceedings of the National Conference on Artificial Intelligence, vol. 20, p. 121. Menlo Park, CA; Cambridge, MA; London; AAAI Press; MIT Press; 1999, 2005.

35. Jamali, M., Ester, M.: Trustwalker: a random walk model for combining trust-based and item-based recommendation. In: Proceedings of the 15 th ACM SIGKDD international conference on Knowledge discovery and data mining, pp. 397-406. ACM, 2009.

36. Crandall, D., Cosley, D., Huttenlocher, D., Kleinberg, J., Suri, S.: Feedback effects between similarity and social influence in online communities. In: Proceedings of the $14^{\text {th }}$ ACM SIGKDD international conference on Knowledge discovery and data mining, pp. 160-168. ACM, 2008.

37. Dunlavy, D., Kolda, T., Acar, E.: Temporal link prediction using matrix and tensor factorizations. ACM Transactions on Knowledge Discovery from Data (TKDD) 5(2), 10, 2011.

38. Menon, A., Elkan, C.: Link prediction via matrix factorization. Machine Learning and Knowledge Discovery in Databases pp. 437-452, 2011.
39. Au Yeung, C., Iwata, T.: Strength of social influence in trust networks in product review sites. In: Proceedings of the fourth ACM international conference on Web search and data mining, pp. 495-504. ACM, 2011.

40. Hong, L., Doumith, A.S., Davison, B .D. Cofactorization machines: modeling user interests and predicting individual decisions in twitter. In: Proceedings of the sixth ACM international conference on Web search and data mining, pp. 557-566. ACM (2013)

41. Jamali, M., Ester, M.: A matrix factorization technique with trust propagation for recommendation in social networks. In: Proceedings of the fourth ACM conference on Recommender systems, pp. 135-142. ACM, 2010.

42. Ma, H., King, I., Lyu, M.R.: Learning to recommend with social trust ensemble. In: Proceedings of the 32nd international ACM SIGIR conference on Research and development in information retrieval, pp. 203-210. ACM, 2009.

43. Noel, J., Sanner, S., Tran, K.N., Christen, P., Xie, L., Bonilla, E.V., Abbasnejad, E., Della Penna, N.: New objective functions for social collaborative filtering. In: Proceedings of the 21st international conference on World Wide Web, pp. 859-868. ACM, 2012.

44. Symeonidis, P., Tiakas, E., Manolopoulos, Y.: Product recommendation and rating prediction based on multi-modal social networks. In: Proceedings of the fifth ACM conference on Recommender systems, pp. 61-68. ACM, 2011.

45. Tang, J., Gao, H., Hu, X., Liu, H.: Exploiting homophily effect for trust prediction. In: Proceedings of the sixth ACM international conference on Web search and data mining, pp. 53-62. ACM, 2013.

46. Tang, J., Gao, H., Liu, H.: mTrust: Discerning multifaceted trust in a connected world. In: Proceedings of the fifth ACM international conference on Web search and data mining, pp. 93-102. ACM, 2012.

47. Tang, J., Gao, H., Liu, H., Das Sarma, A.: eTrust: Understanding trust evolution in an online world. In: Proceedings of the 18th ACM SIGKDD international conference on Knowledge discovery and data mining, pp. 253-261. ACM, 2012.

48. Vasuki, V., Natarajan, N., Lu, Z., Dhillon, I.S.: Affiliation recommendation using auxiliary networks. In: Proceedings of the fourth ACM conference on Recommender systems, pp. 103-110. ACM, 2010.

49. Yang, S.H., Long, B., Smola, A., Sadagopan, N., Zheng, Z., Zha, H.: Like like alike: joint friendship and interest propagation in social networks. In: Proceedings of the $20^{\text {th }}$ international conference on World wide web, pp. 537-546. ACM, 2011.

50. Yuan, Q., Zhao, S., Chen, L., Liu, Y., Ding, S., Zhang, $\mathrm{X}$., Zheng, W.: Augmenting collaborative recommender by fusing explicit social relationships. In: Workshop on Recommender Systems and the Social Web, Recsys, 2009. 
51. Xiang, R., Neville, J., Rogati, M.: Modeling relationship strength in online social networks. In: Proceedings of the 19th international conference on World wide web, 2010.

52. D. Jin, X. Wang, D. He, J. Dang, and W. Zhang, Robust detection of link communities with summary description in social networks, IEEE Trans. Knowl. Data Eng., vol. 33, no. 6, pp. 2737-2749, 2021.

53. Y. Wu, D. Lian, Y. Xu, L. Wu, and E. Chen, Graph convolutional networks with Markov random field reasoning for social spammer detection, in Proceedings of AAAI, pp. 1054-1061, 2020.

54. J. Liu, G. Ma, F. Jiang, C. Lu, P. S. Yu, and A. B. Ragin, Community-preserving graph convolutions for structural and functional joint embedding of brain networks, in Proceedings of BigData, pp. 11631168, 2019.

55. D. Jin, R. Li, and J. Xu, Multiscale community detection in functional brain networks constructed using dynamic time warping, IEEE Trans. Neural Syst. Rehabil. Eng., vol. 28, no. 1, pp. 52-61, 2020.

56. uora: Why does the startup idea of social recommendations consistently fail? In: http://www.quora.com/Why-does-the-startup-idea-ofsocial-recommendationsconsistently- fail (2012)

57. Granovetter, M.: The strength of weak ties: A network theory revisited. American Journal of Sociological theory 1(1), 201-233, 1983.

58. Tang, L., Liu, H.: Relational learning via latent social dimensions. In: Proceedings of the 15th ACM SIGKDD international conference on Knowledge discovery and data mining, pp. 817-26, 2009.

59. Sun, Y., Han, J.: Mining heterogeneous information networks: Principles and methodologies. Synthesis Lectures on Data Mining and Knowledge Discovery 3(2), 1-15, 2012.

60. Levin, D.Z., Cross, R.: The strength of weak ties you can trust: The mediating role of strust in effective knowledge transfer. Management science 50(11), 1477-1490, 2004.

61. Gao, H., Tang, J., Liu, H.: gscorr: Modeling geo-social correlations for new check-ins on location-based social networks. In: Proceedings of the 21st ACM international conference on Information and knowledge management, pp. 1582-1586. ACM, 2012.

62. Ding, Y., Li, X.: Time weight collaborative filtering. In: Proceedings of the 14th ACM international conference on Information and knowledge management, pp. 485-492. ACM, 2005.

63. Koren, Y.: Collaborative filtering with temporal dynamics. In: Proceedings of the $15^{\text {th }}$ ACM SIGKDD international conference on Knowledge discovery and data mining, pp. 447-456. ACM, 2009.

64. Abbassi, Z., Aperjis, C., Huberman, B.A.: Friends versus the crowd: tradeoffs and dynamics. HP Report (2013)

65. Ma, H., Lyu, M.R., King, I.: Learning to recommend with trust and distrust relationships. In: Proceedings of the third ACM conference on Recommender systems, pp. 189-196. ACM, 2009.

66. Cho, J.: The mechanism of trust and distrust formation and their relational outcomes. Journal of Retailing 82(1), 25-35, 2006

67. Narayanan, A., Shmatikov, V.: De-anonymizing social networks. In: Security and Privacy, 2009 30th IEEE Symposium on, pp. 173-187. IEEE, 2009.

68. Liu, J., Zhang, F., Song, X., Song, Y.I., Lin, C.Y., Hon, H.W.: What's in a name?: an unsupervised approach to link users across communities. In: Proceedings of the sixth ACM international conference on Web search and data mining, pp. 495-504. ACM, 2013. 